Bulletin of the Section of Logic

Volume 44:3/4 (2015), pp. 95-110

http://dx.doi.org/10.18778/0138-0680.44.3.4.01

Jānis Cīrulis

\title{
SIMPLE LOGICS FOR BASIC ALGEBRAS
}

\begin{abstract}
An MV-algebra is an algebra $(A, \oplus, \neg, 0)$, where $(A, \oplus, 0)$ is a commutative monoid and $\neg$ is an idempotent operation on $A$ satisfying also some additional axioms. Basic algebras are similar algebras that can roughly be characterised as nonassociative (hence, also non-commutative) generalizations of MV-algebras. Basic algebras and commutative basic algebras provide an equivalent algebraic semantics in the sense of Blok and Pigozzi for two recent logical systems. Both are Hilbert-style systems, with implication and negation as the primitive connectives. We present a considerably simpler logic, $L_{B}$, for basic algebras, where implication and falsum are taken as primitives. We also consider some subvarieties of basic algebras known in the literature, discuss classes of implicational algebras term-equivalent to each of these varieties, and construct axiomatic extensions of $L_{B}$ for which these classes serve as equivalent algebraic semantics.
\end{abstract}

\section{Introduction}

A basic algebra is an algebra $(A, \oplus, \neg, 0)$ of type $(2,1,0)$ satisfying the identities

$\mathrm{B} 1: x \oplus 0=x$,

$\mathrm{B} 2: \neg \neg x=x$,

B3: $\neg(\neg x \oplus y) \oplus y=\neg(\neg y \oplus x) \oplus x$,

$\mathrm{B} 4: \neg(\neg(\neg(x \oplus y) \oplus y) \oplus z) \oplus(x \oplus z)=\neg 0$,

see $[7,8]$; the initial set of axioms (containing six equations and an additional constant 1) turned out to be redundant and was shortened in [9]. 
Basic algebras were introduced as a common generalization of orthomodular lattices and MV-algebras. A basic algebra is an MV-algebra if and only if it is associative, i.e., the operation $\oplus$ is associative (Theorem 8.5.10 in [7]), and (term-equivalent to) an orthomodular lattice if and only if it satisfies identity B6 which will be introduced in Section 4. One more variety of basic algebras which has been intensively studied is that of commutative basic algebras, i.e., those where the operation $\oplus$ is commutative. In particular, every MV-algebra is a commutative basic algebra. For more information on basic algebras, including applications, see the survey papers $[3,5,10]$. We denote the variety of all basic algebras by $\mathbf{B}$, and its subvariety of commutative basic algebras, by CB.

In [1], a non-associative version $L_{C B A}$ of Łukasievicz logic was proposed; it was also shown there that the class of commutative basic algebras provides for $L_{C B A}$ an equivalent algebraic semantics in the sense of [2]. Another system $\mathcal{L}_{B}$, for general basic algebras, was presented in [4]. Both are Hilbert-style systems based on the propositional language with connectives $\rightarrow$ (implication) and $\neg$ (negation). The logic $L_{C B A}$ has five axiom schemes and two rules; $\mathcal{L}_{B}$ has even seven axiom schemes and four rules. In both papers, the main result states that the variety under consideration (i.e., $\mathbf{C B}$ or $\mathbf{B}$ ) induces an algebraic semantics for the corresponding logic, and is proved by checking that the class of implicational algebras determined by the logic is term-equivalent to the respective variety. We point out in this connection a small gap in the proof of Theorem 2 in [1]: it is demonstrated there, in particular, that the variety of basic algebras is interpretable in the constructed equivalent algebraic semantics $K^{*}$ for $L_{C B A}$ (which therefore is a class of $(\rightarrow, \neg)$-algebras), while these are commutative basic algebras which should be so interpreted.

The two logical systems are not explicitly related with each other. Our aim in this paper is to improve the situation: we present another (simpler) logical system $L_{B}$, with $\rightarrow$ and 0 (falsum) as the primitives, for the variety $\mathbf{B}$ of basic algebras; the system is then extended to a logic for $\mathbf{C B}$ adding an appropriate axiom scheme. Moreover, we obtain in the same way logics for several other particular subvarieties of B. Replacement of negation by falsum makes possible to relate the implicational algebras determined by our logics to various appropriate negationless implicational algebras already known in the literature; this enables shortening of several proofs. In particular, the equivalent algebraic semantics of $L_{B}$ turns out to be a class of algebras already knowingly equivalent to $\mathbf{B}$. 
The underlying language of all logical systems considered below is the set of propositional formulas Frm, built from variables by a binary connective $\rightarrow$ and a nullary connective 0 . Other logical connectives can be introduced by the following definitions:

$$
\neg \phi:=\phi \rightarrow 0, \quad \phi \vee \psi:=(\phi \rightarrow \psi) \rightarrow \psi, \quad \phi \& \psi:=\neg(\neg \phi \vee \neg \psi) ;
$$

however, they will not be used. The logic $L_{B}$ is determined by the axiom schemes

A1: $\phi \rightarrow(\psi \rightarrow \phi)$,

A2: $(\phi \rightarrow((\phi \rightarrow \psi) \rightarrow \psi)$,

A3: $((\phi \rightarrow \psi) \rightarrow \psi) \rightarrow((\psi \rightarrow \phi) \rightarrow \phi)$,

A4: $0 \rightarrow \phi$,

and the rules

$$
\begin{aligned}
& \mathrm{R} 1: \frac{\phi, \phi \rightarrow \psi}{\psi}, \\
& \mathrm{R} 2: \frac{\phi \rightarrow \psi}{(\psi \rightarrow \chi) \rightarrow(\phi \rightarrow \chi)}, \\
& \mathrm{R} 3: \frac{\phi \rightarrow \psi, \psi \rightarrow \phi}{(\chi \rightarrow \phi) \rightarrow(\chi \rightarrow \psi)} .
\end{aligned}
$$

We describe in the next section a class (in fact, a variety) $\mathbf{I B}_{0}$ of $(\rightarrow, 0)$ algebras which is term-equivalent to the variety of basic algebras, and show in Section 3 that the logic $L_{B}$ is algebraizable and $\mathbf{I B}_{0}$ is its equivalent algebraic semantics. In Section 4 , we discuss several known subvarieties of B (in particular, CB) and construct appropriate axiomatic extensions of $L_{B}$ for what they serve as equivalent semantics.

\section{Some related classes of implicational algebras}

It was proved in [8] that the class of basic algebras is term-equivalent to a variety of algebras $(A, \rightarrow, 0,1)$ of type $(2,1,1)$, where $\rightarrow$ is interpreted as an implication-like operation. The corresponding operation definitions are (see Theorem 3.10 and Definition 3.2 of [8]):

$$
x \rightarrow y:=\neg x \oplus y, 1:=\neg 0 \text { and } x \oplus y:=(x \rightarrow 0) \rightarrow y, \neg x:=x \rightarrow 0 .
$$


Later, the equational axioms of this variety were simplified and the constant 1 was shown to be definable in terms of implication:

I0: $x \rightarrow x=1$.

The $\rightarrow$-reducts of algebras from the variety were named implication basic algebras and characterised by the following axioms:

I1: $(x \rightarrow x) \rightarrow x=x$,

I2: $(x \rightarrow y) \rightarrow y=(y \rightarrow x) \rightarrow x$,

I3: $(((x \rightarrow y) \rightarrow y) \rightarrow z) \rightarrow(x \rightarrow z)=x \rightarrow x$.

[11, Definition 1]; see also Definition 7.1 in [5]. Let us denote here the variety of these algebras by IB. Owing to I0, we may consider them, where convenient, also as $(\rightarrow, 1)$-algebras.

The relation $\leq$ on an $\mathbf{I B}$-algebra $A$ defined by

$$
x \leq y \text { iff } x \rightarrow y=1
$$

is a partial order on $A$. Thus, an element 0 is the least element in $A$ if and only if the following holds for any $x$ :

I4: $0 \rightarrow x=1$;

in particular, such an element is unique when it exists. We denote by $\mathbf{I B}_{0}$ the class of bounded IB-algebras $(A, \rightarrow, 0)$ (they were called implication basic algebras with the least element in $[11,5])$. It is in fact a variety, characterised, besides I1-I3, by a version $0 \rightarrow x=x \rightarrow x$ of I4. Of course, $\mathbf{I B}_{0}$-algebras may be treated also as algebras in the signature $(\rightarrow, 0,1)$ satisfying I0-I4. As mentioned above, the variety $\mathbf{I B}_{0}$ is term-equivalent to that of basic algebras (cf. [11, Theorem 6]). Another system of three equations for $\mathbf{I B}_{0}$ can be obtained, using (2.1), directly from B1-B4 (notice that the translations of $\mathrm{B} 1$ and $\mathrm{B} 2$ are the same); it consists of $\mathrm{I} 2$, a variant of I3 and

I5: $(x \rightarrow 0) \rightarrow 0)=x ;$

see, e.g., Section 3 in [5] (or [6], where further reductions are considered).

Thus, the class $\mathbf{I B}_{0}$ is characterised by equational axioms. For our purposes in the next section, $\leq$-based axioms, i.e., equations of the form $\phi=1$ and quasi-equations involving only equalities of this kind will be more useful. It is remarked in [14] (pp. 8 and 14) that there is a close connection between implication basic algebras and commutative wBCK* algebras in the sense of $[12,16]$, i.e., order duals of commutative weak BCK-algebras of $[14,16]$. A $w B C K^{*}$-algebra was defined in [12] to be an 
algebra $A:=(A, \rightarrow, 1)$ such that $A$ is a poset with the greatest element 1 and $\rightarrow$ is a binary operation on $A$ satisfying (2.2) and a weakened form of the exchange law:

$$
\text { if } x \leq y \rightarrow z \text {, then } y \leq x \rightarrow z .
$$

A wBCK* algebra is said to be commutative if it satisfies the identity I2. The class of commutative wBCK*-algebras is a variety, which, according to [14, Theorem 3.4] is characterised by axioms I0, I2 and the following versions of $\mathrm{I} 1$ and $\mathrm{I} 3$ :

I1': $1 \rightarrow x=x$,

I3': $(((y \rightarrow x) \rightarrow x) \rightarrow z) \rightarrow(x \rightarrow z)=1$,

provided the order is defined as in (2.2); [14, Theorem 3.5] shows that the variety is actually three-based. Evidently, this variety coincides with the variety of implication basic algebras considered as algebras $(A, \rightarrow, 1)$ satisfying I0.

A bounded wBCK* $^{*}$-algebra is one possessing the least element 0 . Such a wBCK*-algebra $A$ always satisfies $\mathrm{I} 4$ and may therefore be considered also as an algebra $(A, \rightarrow, 0,1)$. Then $A$ may be regarded as $\mathbf{I B}_{0}$-algebra, and conversely. We thus have arrived at the following observation.

Proposition 1. The classes (varieties) of $\boldsymbol{I B}_{0}$-algebras and bounded commutative $w B C K^{*}$-algebras coincide.

\section{The logic $L_{B}$}

We first adapt to $L_{B}$ the general concepts of equivalent algebraic semantics and algebraizable logic (Definitions 2.8 and 2.10 in [2]). It will be convenient, when dealing with algebras of kind $(A, \rightarrow, 0)$, to consider formulas from Frm also as terms and build from them equations $\phi=\psi$.

Given a class $K$ of such algebras, the $K$-consequence relation $\models_{K}$ (between sets of equations and equations) is defined in the usual way (cf. $[2])$ : if $\Gamma$ is a set of equations, then

$\Gamma \models_{K} \phi=\psi$ if and only if the equation $\phi=\psi$ holds under any interpretation in an algebra from $K$ whenever all equations from $\Gamma$ hold under the same interpretation.

In an algebraic context, we also write 1 for the term $0 \rightarrow 0$ and $\phi \leq \psi$ for the equation $\phi \rightarrow \psi=1$, where $\phi$ and $\psi$ are arbitrary terms. We thus may 
also consider 1 as a symbol for the constant $0 \rightarrow 0$ in an algebra $A$, and $\leq$ as a symbol for the binary relation defined on $A$ by the family of equations $\phi \rightarrow \psi=1$.

Since only one fixed defining equation $p=1$ and only one fixed set $\{p \rightarrow q, q \rightarrow p\}$ of equivalence formulas will be necessary here, we do not mention them explicitly in the subsequent specialisation of the general definition [2, Definition 2.8].

A class $K$ of algebras $(A, \rightarrow, 0)$ is said to be an equivalent algebraic semantics for $L_{B}$ if, for all $\Gamma \subseteq$ Frm and $\phi, \psi \in F r m$,

$$
\begin{aligned}
& \Gamma \vdash \phi \text { if and only if }\{\psi=1: \psi \in \Gamma\} \models_{K} \phi=1, \\
& \phi \rightarrow \psi=1=\psi \rightarrow \phi \models_{K} \phi=\psi \text { and conversely. }
\end{aligned}
$$

The logic $L_{B}$ is said to be algebraizable if it admits an equivalent algebraic semantics.

REMARK 1. Like any algebraizable logical system, $L_{B}$ may have various equivalent algebraic semantics. By Corollaries 2.15 and 2.11 of [2], they generate the same quasivariety, which is another equivalent algebraic semantics for $L_{B}$. If classes $K$ and $K^{\prime}$ are both equivalent algebraic semantics, then they, strictly speaking, may have different defining equations and different equivalence formulas. However, both sets of equivalence formulas are deductively equivalent in $L_{B}$, and every defining equation of one semantics is both a $K$-consequence and a $K^{\prime}$-consequence of the set of all defining equations of the other semantics (Corollary 2.15 in [2]). Therefore, the formally restricted notion of algebraizability (since related to the defining equation and equivalence formulas fixed above) is still adequate.

Lemma 2. The logic $L_{B}$ is algebraizable.

Proof: According to [2, Theorem 4.7], the particular logical system $L_{B}$ is algebraizable provided the following statements hold in it for all formulas $\phi, \psi, \chi$ :

(i) $\vdash \phi \rightarrow \phi$,

(ii) $\phi \rightarrow \psi \vdash \phi \rightarrow \psi$,

(iii) $\phi \rightarrow \psi, \psi \rightarrow \chi \vdash \phi \rightarrow \chi$,

(iv-1) $\phi \rightarrow \psi, \psi \rightarrow \phi, \phi^{\prime} \rightarrow \psi^{\prime}, \psi^{\prime} \rightarrow \phi^{\prime} \vdash\left(\phi \rightarrow \phi^{\prime}\right) \rightarrow\left(\psi \rightarrow \psi^{\prime}\right)$,

(iv-2) $\phi \rightarrow \psi, \psi \rightarrow \phi, \phi^{\prime} \rightarrow \psi^{\prime}, \psi^{\prime} \rightarrow \phi^{\prime} \vdash\left(\psi \rightarrow \psi^{\prime}\right) \rightarrow\left(\phi \rightarrow \phi^{\prime}\right)$, 
$(\mathrm{v}-1) \phi \vdash \phi \rightarrow(0 \rightarrow 0)$,

$(\mathrm{v}-2) \phi \vdash(0 \rightarrow 0) \rightarrow \phi$,

(v-3) $(0 \rightarrow 0) \rightarrow \phi \vdash \phi$.

We prove that this is indeed the case.

(ii) Trivial.

(iii) By R1 from R2.

(i) First observe that R2, A2 and (iii) yield the derivation

$$
\phi \rightarrow(\psi \rightarrow \chi),((\psi \rightarrow \chi) \rightarrow \chi) \rightarrow(\phi \rightarrow \chi), \psi \rightarrow((\psi \rightarrow \chi) \rightarrow \chi), \psi \rightarrow(\phi \rightarrow \chi)
$$

of a sequent

$$
\phi \rightarrow(\psi \rightarrow \chi) \vdash \psi \rightarrow(\phi \rightarrow \chi) .
$$

Now A4, A1, (3.3) and R1 yield a derivation for $\phi \rightarrow \phi$ :

$$
0 \rightarrow 0, \phi \rightarrow((0 \rightarrow 0) \rightarrow \phi),(0 \rightarrow 0) \rightarrow(\phi \rightarrow \phi), \phi \rightarrow \phi .
$$

(iv-1) We have:

$$
\begin{aligned}
& \psi \rightarrow \phi \vdash\left(\phi \rightarrow \phi^{\prime}\right) \rightarrow\left(\psi \rightarrow \phi^{\prime}\right) \quad \text { by R2, } \\
& \phi^{\prime} \rightarrow \psi^{\prime}, \psi^{\prime} \rightarrow \phi^{\prime} \vdash\left(\psi \rightarrow \phi^{\prime}\right) \rightarrow\left(\psi \rightarrow \psi^{\prime}\right) \quad \text { by R3, }
\end{aligned}
$$$$
\left(\phi \rightarrow \phi^{\prime}\right) \rightarrow\left(\psi \rightarrow \phi^{\prime}\right),\left(\psi \rightarrow \phi^{\prime}\right) \rightarrow\left(\psi \rightarrow \psi^{\prime}\right) \vdash
$$$$
\left(\phi \rightarrow \phi^{\prime}\right) \rightarrow\left(\psi \rightarrow \psi^{\prime}\right) \text { by (iii). }
$$

From these sequents, (iv-1) easily follows.

(iv-2) This is a variant of (iv-1).

(v-1) A4, A1 and R1 give us a required derivation

$$
\phi, 0 \rightarrow 0,(0 \rightarrow 0) \rightarrow(\phi \rightarrow(0 \rightarrow 0)), \phi \rightarrow(0 \rightarrow 0) .
$$

(v-2) By A1 and R1.

(v-3) By (i) and R1.

We now state the main result of the paper.

THEOREM 3. The variety $\boldsymbol{I B}_{0}$ is an equivalent algebraic semantics for $L_{B}$.

Proof: It follows from [2, Theorem 2.17] and Lemma 2 that the class $K$ of algebras $(A, \rightarrow, 0)$ defined by the following equations and quasi-equations is an equivalent algebraic semantics for $L_{B}$ :

(i-1) $x \leq(y \rightarrow x)$,

(i-2) $x \leq(x \rightarrow y) \rightarrow y$, 
(i-3) $(x \rightarrow y) \rightarrow y \leq(y \rightarrow x) \rightarrow x$,

(i-4) $0 \leq x$,

(ii) $x \leq x$,

(iii-1) if $x=1$ and $x \leq y$, then $y=1$,

(iii-2) if $x \leq y$, then $y \rightarrow z \leq x \rightarrow z$,

(iii-3) if $x \leq y$ and $y \leq x$, then $z \rightarrow x \leq z \rightarrow y$,

(iv) if $x \leq y$ and $y \leq x$, then $x=y$.

We have to prove that $K=\mathbf{I B}_{0}$.

At first, notice that, due to Lemma 2(iii), (3.3) and (3.1), the following quasi-equations also hold in $K$ :

$(\mathrm{v}-1)$ if $x \leq y$ and $y \leq z$, then $x \leq z$,

$(\mathrm{v}-2)$ if $x \leq y \rightarrow z$, then $y \leq x \rightarrow z \quad$ (this is the law (2.3)).

Now, (ii), (v-1), (iv) and (i-4) show that the relation $\leq$ on an algebra $A$ from $K$ is an order with the least element 0 , while (iii- 1 ) says that 1 is its maximal element. Actually, (v-2) and (i-1) entail $y \leq 0 \rightarrow 0$, which means that 1 is the greatest element in $A$. Therefore, all algebras in $K$ are bounded posets, and (iii-3) becomes trivially true.

Further, (v-2) and the equivalence (2.2), which immediately follows from the above conventions concerning 1 and $\leq$, show that members of $K$ are even bounded wBCK*-algebras. It follows from (i-3) that wBCK*algebras in $K$ are commutative. Thus, $K \subseteq \mathbf{I B}_{0}$ by Proposition 1 .

Conversely, every $\mathbf{I B}_{0}$-algebra belongs to $K$. To see this, assume that $A$ is such an algebra. By Proposition 1, then $A$ is a bounded commutative wBCK*-algebra. This implies that $A$ satisfies (2.2) and, hence, also (i-1), (i-3), (i-4), (ii), (iii-1), (iii-3) and (iv). Moreover, it is easily seen that both (i-2) and (iii-2) hold in any wBCK-algebra (cf. [12, Lemma 2(a,b)] or [16, Proposition 2.1]). Thus, $\mathbf{I B}_{0} \subseteq K$.

\section{Some subvarieties of B and their logics}

As shown in Propositions 3.5-3.7 of [8], one can define lattice operations in every basic algebra $A$ so that it becomes a bounded lattice in which the involution $\neg$ is antitone, i.e., acts as the so called De Morgan complementation (m-complementation, for short). In particular,

$$
x \vee y:=\neg(\neg x \oplus y) \oplus y, \quad x \wedge y:=\neg(\neg x \vee \neg y), \quad 1:=\neg 0,
$$


and, in the $\mathbf{I B}_{0}$-algebra corresponding to $A$,

$$
(x \rightarrow y) \rightarrow y=x \vee y=(y \rightarrow x) \rightarrow x .
$$

The lattice order in $A$ is characterised by

$$
x \leq y \text { iff } \neg x \oplus y=1 .
$$

If the induced lattice is orthocomplemented, i.e., satisfies the identity

$$
\neg x \vee x=1,
$$

or orthomodular, i.e., is orthocomplemented and satisfies the condition

$$
\text { if } x \leq y \text { and } x \vee \neg y=1 \text {, then } x=y \text {, }
$$

we say that such is the basic algebra $A$ itself. This is the case if and only if $A$ satisfies the first, respectively, the second of the following identities:

B5: $x \oplus x=x$,

B6: $y \oplus(x \wedge y)=y$;

see [8, Theorem 3.13] or [5, Corollary 4.2], resp., [3, Theorem 6] or [5, Theorem 4.3]. The identity B6 is a converted form of the equivalent quasiidentity

B7: if $x \leq y$, then $y \oplus x=y$.

The lattice induced in a basic algebra is even sectionally m-complemented, i.e., has m-complemented sections: for every $p$ and all $x \geq p$, the element $\neg x \oplus p$ (i.e., $x \rightarrow p$ ) is an m-complement of $x$ in the upper section $[p, 1]$ - see Section 3 in [8]. If each sectional m-complement is actually an orthocomplement, we call such a basic algebra sectionally orthocomplemented. These basic algebras are characterised by an additional identity

B8: $x \oplus(x \oplus y)=x \oplus y$,

see Corollary 5 below. Notice that an orthocomplemented basic algebra is not necessary sectionally orthocomplemented in this sense. It is easily seen, however, that every section of a sectionally orthocomplemented basic algebra turns out to be orthomodular if and only if the algebra itself is orthomodular.

At last, a basic algebra is called monotone (also left-monotone), if the following law is fulfilled in it:

B9: if $x \leq y$, then $z \oplus x \leq z \oplus y$; 
see, e.g., [5, Definition 2.20]. As the right monotonicity law is fulfilled in all basic algebras ([8, Proposition 3.5(b)]), every commutative basic algebra is monotone.

Let the symbols $\mathbf{C B}, \mathbf{A B}, \mathbf{O B}, \mathbf{s O B}, \mathbf{O m B}, \mathbf{M B}$ stand for the class of commutative, associative, orthocomplemented, sectionally orthocomplemented, orthomodular, and monotone basic algebras, respectively (of course, $\mathbf{A B}$ is the class of all $\mathrm{MV}$-algebras). We now move to the respective subvarieties of $\mathbf{I B}_{0}$.

THEOREM 4. A basic algebra is commutative, associative, orthocomplemented, sectionally orthocomplemented, orthomodular or monotone if and only if its associated $\mathbf{I B}_{0}$-algebra satisfies the (quasi)identity (a), (b), (c), (d), (e) or (f), respectively:

(a) $(x \rightarrow 0) \rightarrow y \leq(y \rightarrow 0) \rightarrow x$

(b) $x \rightarrow y \leq(z \rightarrow x) \rightarrow(z \rightarrow y)$,

(c) $(x \rightarrow 0) \rightarrow x \leq x$,

(d) $x \rightarrow(x \rightarrow y) \leq x \rightarrow y$,

(e) if $x \leq y$, then $(y \rightarrow 0) \rightarrow x=y$,

(f) $x \rightarrow y \leq x \rightarrow((z \rightarrow y) \rightarrow y)$.

The condition (e) is equivalent, in every basic algebra, to the conjunction of (c) and

(g) $((x \rightarrow y) \rightarrow y) \rightarrow 0 \leq y \rightarrow x$.

Proof: (a) In virtue of (2.1), the conjunction of (a) and its reverse inequality is the implicative counterpart of the commutative law for $\oplus$.

(b) By [8, Proposition 3.11], a basic algebra is an MV-algebra iff (b) holds. See also the note subsequent to Proposition 1 in [4].

(c) Suppose that $A$ is a basic algebra. By (2.1) and (4.1), the condition (c) means that $1=(\neg x \rightarrow x) \rightarrow x=\neg x \vee x$, i.e., that $A$ is orthocomplemented (and conversely).

(d) According to Corollary 3.5 of [16], a wBCK*-algebra is sectionally orthocomplemented if and only if it satisfies the condition

$$
x \leq y \text { whenever } x \leq x \rightarrow y .
$$

By [14, Lemma 4.1], the condition is equivalent in commutative wBCK*algebras to the contraction law 


$$
x \rightarrow(x \rightarrow y)=x \rightarrow y
$$

(alternatively, see Corollaries 6.5 and 6.6 in [14]). It is further equivalent to the inequality (d), as the reverse inequality of the latter holds in every $\mathrm{wBCK}^{*}$-algebra by the condition (i-1) of Theorem 3 . The conclusion follows now from Proposition 1.

(e) This condition is the counterpart of B7.

(f) Those wBCK*-algebras satisfying the isotonicity law

$$
\text { if } x \leq y \text {, then } z \rightarrow x \leq z \rightarrow y
$$

were termed quasi-BCK${ }^{*}$-algebras or just $q B C K^{*}$-algebras in [13]. Evidently, this law is the counterpart of B9. According to [14, Proposition 3.6], a wBCK*-algebra is a $\mathrm{qBCK}^{*}$-algebra if and only (f) holds in it.

As to the last assertion of the theorem, a basic algebra $A$ satisfying (c) is orthocomplemented; let us see that $(\mathrm{g})$ makes it orthomodular. In view of (4.2), suppose that $x \leq y$ and $x \vee \neg y=1$, i.e.,

$$
(x \rightarrow y) \rightarrow y=x \vee y=y \text { and } \neg y \rightarrow x \leq x
$$

- see (4.1). By (g) and the definition of $\neg$ in (2.1), then $\neg y \leq y \rightarrow x$; this implies, by the exchange rule (2.3) (which holds in the $\mathbf{I B}_{0}$-algebra associated to $A$ ), that $y \leq \neg y \rightarrow x$, whence $y \leq x$ and $x=y$. Thus, $A$ is indeed orthomodular; therefore, (c) and (g) together imply (e).

Conversely, (c) is an easy consequence of (e). Further, due to the exchange rule, (e) implies that $y \rightarrow 0 \leq y \rightarrow x$ whenever $x \leq y$. In particular, as $x \leq(y \rightarrow x) \rightarrow x$ (recall (4.1)),

$$
((y \rightarrow x) \rightarrow x) \rightarrow 0 \leq((y \rightarrow x) \rightarrow x) \rightarrow x \leq y \rightarrow x ;
$$

the latter inequality can be easily verified using item (iii-2) of Theorem 3 . So, (g) also follows from (e).

Notice that, due to I5, (a) can be rewritten as the contraposition law $(x \rightarrow y) \leq(y \rightarrow 0) \rightarrow(x \rightarrow 0)$, and, due to condition (i-1) of Theorem 3 , (c) can be replaced by the identity $(x \rightarrow 0) \rightarrow x=x$. This identity, as the definition of $\oplus$ in (2.1) shows, is the the implicational form of the idempotency law $x \oplus x=x$. Also, due to I2 and (2.2), (c) is equivalent to $x \rightarrow(x \rightarrow 0) \leq x \rightarrow 0$, a particular case of $(\mathrm{d})$. As MV-algebras are known to be equivalent to bounded BCK-algebras [17], an $\mathbf{I B}_{0}$-algebra satisfies (b) if and only if it is a BCK*-algebra. By [14, Proposition 3.6], (f) is 
equivalent to a shorter axiom $x \rightarrow y \leq x \rightarrow(z \rightarrow y)$. At last, since (4.4) is an implicational counterpart of B8 (recall I5), we immediately arrive at the following conclusion.

COROLlary 5. The class of sectionally orthocomplemented basic algebras is a variety characterised by $\mathrm{B} 1-\mathrm{B} 4$ and $\mathrm{B} 8$.

A few other subvarieties of $\mathbf{B}$ are reviewed in [6].

Now consider the following formula schemes suggested by items (a)-(d), (f) and (g) of the preceding theorem:

A5: $((\phi \rightarrow 0) \rightarrow \psi) \rightarrow((\psi \rightarrow 0) \rightarrow \phi)$,

A6: $(\phi \rightarrow \psi) \rightarrow((\chi \rightarrow \phi) \rightarrow(\chi \rightarrow \psi))$,

A7: $((\phi \rightarrow 0) \rightarrow \phi) \rightarrow \phi$,

A8: $(\phi \rightarrow(\phi \rightarrow \psi)) \rightarrow(\phi \rightarrow \psi)$,

A9: $(((\phi \rightarrow \psi) \rightarrow \psi) \rightarrow 0) \rightarrow(\psi \rightarrow \phi)$,

A10: $(\phi \rightarrow \psi) \rightarrow(\phi \rightarrow((\chi \rightarrow \psi) \rightarrow \psi))$.

Using them, we introduce logical systems for the subvarieties of $\mathbf{B}$ characterised in Theorem 4 as follows:

$L_{C B}$ to be the extension of $L_{B}$ by A5 (for CB), $L_{A B}$ to be the extension of $L_{B}$ by $\mathrm{A} 6$ (for $\mathbf{A B}$ ), $L_{O B}$ to be the extension of $L_{B}$ by A7 (for $\mathbf{O B}$ ), $L_{S O B}$ to be the extension of $L_{B}$ by A8 (for $\mathbf{s O B}$ ),

$L_{O m B}$ to be the extension of $L_{O B}$ by A9 (for $\mathbf{O m B}$ ), $L_{M B}$ to be the extension of $L_{B}$ by A10 (for $\mathbf{M B}$ ).

As all these logics are extensions of $L_{B}$, the subsequent corollary follows immediately from Lemma 2 (cf. [2, Corollary 4.9]).

Corollary 6. The logics $L_{C B}, L_{A B}, L_{O B}, L_{s O B}, L_{O M}, L_{O m B}$ and $L_{M B}$ are algebraizable.

Corollary 7. The variety of $\boldsymbol{I B}_{0}$-algebras corresponding to that of commutative (associative, orthocomplemented, sectionally orthocomplemented, orthomodular, monotone) basic algebras is an equivalent algebraic semantic for the logic $L_{C B}$ (resp., $L_{A B}, L_{O B}, L_{s O B}, L_{O m B}, L_{M B}$ ). 
Proof: By Theorem 3 and (3.1), any formula $\chi$ is derivable in $L_{B}$ from a set of formulas $\Gamma+\mathrm{A} 5$ if and only if the equation $\chi=1$ is a $\mathbf{I B}_{0}$-consequence of $\Gamma+\mathrm{A} 5$. Consequently, $\chi$ is derivable in $L_{C B}$ from $\Gamma$ if and only if $\chi=1$ is a $K$-consequence of $\Gamma$, where $K$ is the class of $\mathbf{I B}_{0}$-algebras satisfying the identity (a) in Theorem 4. Of course, (3.2) holds true for this $K$. So, this class is an algebraic semantics for $L_{C B}$.

The other statements are proved similarly.

We end the section with several observations that make it possible to simplify some of the considered logics. Notice that the rule R3 was not required in proofs of (3.3) and items (i)-(iii) of Lemma 2.

Proposition 8. In $L_{M B}$, $\mathrm{A} 10$ and $\mathrm{R} 3$ may be replaced by the rule

$\mathrm{R} 4: \frac{\phi \rightarrow \psi}{(\chi \rightarrow \phi) \rightarrow(\chi \rightarrow \psi)}$

Proof: Evidently, R3 is a consequence of R4. By A1 and R4, the sequence

$$
\phi \rightarrow((\psi \rightarrow \phi) \rightarrow \phi),(\chi \rightarrow \phi) \rightarrow(\chi \rightarrow((\psi \rightarrow \phi) \rightarrow \phi))
$$

is a derivation of A10. Thus, $\left(L_{M B}-\mathrm{A} 10-\mathrm{R} 3+\mathrm{R} 4\right)$ is an extension of $L_{M B}$. On the other hand, the rule $\mathrm{R} 4$, being the counterpart of the isotonicity rule (4.5), is derivable in $L_{M B}$ by virtue of (3.1).

Proposition 9. The rule $\mathrm{R} 3$ is dependent in $L_{C B}$.

Proof: We first show, without using R3, that in $L_{C B}$

(i) $\vdash((\psi \rightarrow 0) \rightarrow(\chi \rightarrow 0)) \rightarrow(\chi \rightarrow \psi)$,

(ii) $\vdash(\chi \rightarrow \phi) \rightarrow((\phi \rightarrow 0) \rightarrow(\chi \rightarrow 0))$.

Derivation for (i):

(a) $((\psi \rightarrow 0) \rightarrow(\chi \rightarrow 0)) \rightarrow(((\chi \rightarrow 0) \rightarrow 0) \rightarrow \psi)$

(b) $((\chi \rightarrow 0) \rightarrow 0) \rightarrow(((\psi \rightarrow 0) \rightarrow(\chi \rightarrow 0)) \rightarrow \psi)$

(c) $\chi \rightarrow((\chi \rightarrow 0) \rightarrow 0)$

(d) $\chi \rightarrow(((\psi \rightarrow 0) \rightarrow(\chi \rightarrow 0)) \rightarrow \psi)$

$[(\mathrm{c}),(\mathrm{b}), \mathrm{L} 2(\mathrm{iii})]$

(e) $((\psi \rightarrow 0) \rightarrow(\chi \rightarrow 0)) \rightarrow(\chi \rightarrow \psi)$ $[(d),(3.3)]$ 
Derivation for (ii):

(a) $(((\chi \rightarrow 0) \rightarrow 0) \rightarrow \phi) \rightarrow((\phi \rightarrow 0) \rightarrow(\chi \rightarrow 0))$

(b) $\chi \rightarrow((\chi \rightarrow \phi) \rightarrow \phi)$

(c) $(0 \rightarrow \chi) \rightarrow(((\chi \rightarrow 0) \rightarrow 0) \rightarrow \chi)$

(d) $((\chi \rightarrow 0) \rightarrow 0) \rightarrow \chi$ $[\mathrm{A} 4,(\mathrm{c}), \mathrm{R} 1]$

(e) $((\chi \rightarrow 0) \rightarrow 0) \rightarrow((\chi \rightarrow \phi) \rightarrow \phi)$ $[(\mathrm{d}),(\mathrm{b}), \mathrm{L} 2(\mathrm{iii})]$

(f) $(\chi \rightarrow \phi) \rightarrow(((\chi \rightarrow 0) \rightarrow 0) \rightarrow \phi)$

(g) $(\chi \rightarrow \phi) \rightarrow((\phi \rightarrow 0) \rightarrow(\chi \rightarrow 0))$

$[(\mathrm{f}),(\mathrm{a}), \mathrm{L} 2(\mathrm{iii})]$

To get R3 in $\left(L_{C B}-\mathrm{R} 3\right)$, it suffices to derive R4:

(a) $\phi \rightarrow \psi$

[hyp]

(b) $(\psi \rightarrow 0) \rightarrow(\phi \rightarrow 0)$

$[(\mathrm{a}), \mathrm{R} 2]$

(c) $((\phi \rightarrow 0) \rightarrow(\chi \rightarrow 0)) \rightarrow((\psi \rightarrow 0) \rightarrow(\chi \rightarrow 0))$

$[(\mathrm{b}), \mathrm{R} 2]$

(e) $(\chi \rightarrow \phi) \rightarrow(\chi \rightarrow \psi)$

[(ii), (c), L2(iii), (i), L2(iii)]

Proposition 10. In $L_{s O B}$, the axioms $\mathrm{A} 3$ and $\mathrm{A} 8$ may be replaced by

A11: $((\phi \rightarrow \psi) \rightarrow \phi) \rightarrow \phi$.

Proof: In view of $\mathrm{A} 3$, axioms $\mathrm{A} 11$ and $\mathrm{A} 8$ are equivalent in $L_{B}$ and, hence, derivable in $L_{s O B}$. We have to prove that $\mathrm{A} 3$ and $\mathrm{A} 8$ are derivable in $\left(L_{B}-\mathrm{A} 3+\mathrm{A} 11\right)$.

According to [16, Corollary 3.5], a wBCK*-algebra $A$ is sectionally orthocomplemented if and only if it is commutative and satisfies the condition (4.3). By [16, Corollary 3.7], this is the case if and only if $A$ satisfies the Pierce law $(x \rightarrow y) \rightarrow x=x$. This concerns, of course, also bounded ${ }_{\text {wBCK}}{ }^{*}$-algebras. Actually, it is sufficient to consider only a half of the law, $(x \rightarrow y) \rightarrow x \leq x$, for the reverse inequality holds in any $\mathrm{wBCK}^{*}$-algebra.

Now it follows that both I2 and (4.3) are consequences, in the class of ${ }_{\text {wBCK}}{ }^{*}$-algebras, of the latter inequality, which is the algebraic counterpart of A11. Notice that the axiom A3 is not used in the proof of Lemma 2, and an inspection of the proof of Theorem 3 shows that the class of bounded ${ }_{\text {wBCK}}{ }^{*}$-algebras is actually an equivalent algebraic semantics of $\left(L_{B}-\right.$ A3). Hence, in view of the corresponding version of (3.1), A3 and A8 are indeed derivable from A11 in $\left(L_{B}-\mathrm{A} 3\right)$. 
Corollary 11. In $L_{O m B}$, the axioms $\mathrm{A} 3$ and $\mathrm{A} 7$ may be replaced by $\mathrm{A} 11$.

\section{Acknowledgments}

This work was supported by Latvian Council of Science, Grant No. 271/2012. The author thanks Ivan Chajda for preprints of [5] and [10], and the anonymous referee, for the careful reading of the text.

\section{References}

[1] M. Botur, R. Halaš, Commutative basic algebras and non-associative fuzzy logics, Arch. Math. Logic, 48 (2009), pp. 243-255.

[2] W.J. Blok, D. Pigozzi, Algebraizable logics, Mem. Amer. Math. no. 296, Providence, Rhode Island (1989).

[3] I. Chajda, Basic algebras and their applications, an overview, in: Czermak, J. (ed.) et al., Klagenfurt: Verlag Johannes Heyn. Contributions to General Algebra, 20 (2012), pp. 1-10.

[4] I. Chajda, The propositional logic induced by means of basic algebras, Int. J. Theor. Phys., 54 (2015), pp. 4306-4312.

[5] I. Chajda, Basic algebras, logics, trends and applications, Asian-Eur. J. Math. 08, 1550040 (2015) [46 pages].

[6] I. Chajda, R. Halaš, On varieties of basic algebras, Soft Comput., 19 (2015), pp. 261-267.

[7] I. Chajda, R. Halaš, J. Kühr, Semilattice Structures, Heldermann Verlag, Lemgo (2007).

[8] I. Chajda, R. Halaš, J. Kühr, Many-valued quantum algebras, Algebra Universalis, 60 (2009), pp. 63-90.

[9] I. Chajda, M. Kolařík, Independence of axiom system of basic algebras, Soft Computing, 13 (2009), 41-43.

[10] I. Chajda, J. Kühr, Basic algebras, RIMS Kokyuroku, Univ. of Kyoto, 1846 (2013), 1-13.

[11] I. Chajda, M. Kolař́k, F. Švrček, Implication and equivalential reducts of basic algebras, Acta Univ. Palacki Olomouc, Fac. rer. nat., Mathematica, 49 (2010), 21-36.

[12] J. Cīrulis, Implication in sectionally pseudocomplemented posets, Acta Sci. Math. (Szeged), 74 (2008), 477-491.

[13] J. Cīrulis, Residuation subreducts of pocrigs, Bull. Sect. Logic (Lodź), 39 (2010), 11-16. 
[14] J. C̄̄rulis, On commutative weak BCK-algebras, arXiv:1304:0999.

[15] J. Cirulis, Quasi-orthomodular posets and weak BCK-algebras, Order 31 (2014), 403-419.

[16] J. Cirulis, On some classes of commutative weak BCK-algebras, Studia Logica, 103 (2015), 479-490.

[17] D. Mundici, $M V$-algebras are categorically equivalent to bounded commutative BCK-algebras, Math. Jap., 31 (1986), 889-894.

Institute of Mathematics and Computer Science

University of Latvia

Raina b., 29, Riga LV-1459, Latvia

email: janis.cirulis@lu.lv 